\title{
FINDING OF POSTCRITICAL STATES OF AN ARCH
}

\author{
M. Kalina*, P. Frantík ${ }^{* *}$

\begin{abstract}
Postcritical solutions of the elastic arched rod model with geometric imperfection are described in the present paper along with associated maps of potential energy surfaces. This contribution explains, how it is possible to find these postcritical states and their occurrence.
\end{abstract}

\section{Keywords: potential energy, bifurcation, geometric imperfection, slender rod model, postcritical state}

\section{Introduction}

When studying slender rod models during loading, an interesting phenomenon can be observed. The model keeping a certain position suddenly turns into a remarkably different position, substantial change of its potential energy takes place. A problem with this phenomenon was first solved by Leonhard Euler in the form of ideal beam buckling problem and further extended by (Timoshenko, 1961). The load required to achieve this phenomenon is called critical and originated states after overstepping this level are so-called postcritical. The phenomenon is accompanied with occurrence of presence of more static equilibrium states and the particular static solution is not unique and even can be unstable (Dinkler, 2006). In general, it may be difficult to find these concurrently existing states and determine which of them will be realized during loading a real structure. For this purpose, was developed a method which can helps with searching for these concurrent states and makes a map of potential energy surface associated with the structure and its equilibrium states (Frantík, 2007).

In this paper we present postcritical solutions and potential energy surfaces of a plane slender arch with geometric imperfections from ideal shape. These results are found and calculated by enhanced method implemented in computer application (MapPoEng, 2017). The research follows our study of behaviour of von Mises truss (Kalina, 2016).

\section{Slender arch}

The arch is loaded by a force in the middle of the span (see Fig. 1) and has cross-section area $A=0.008457 \mathrm{~m}^{2}$, the second area moment $I=1.32 \cdot 10^{-5} \mathrm{~m}^{4}$, the span $L=10 \mathrm{~m}$ and rise $H=2 \mathrm{~m}$. The beginning of coordinate system is oriented in the half of the span of arch, so that the coordinate of the initial node is $x_{0}=-5 \mathrm{~m} ; y_{0}=0 \mathrm{~m}$, and the coordinate of the end node is $x_{0}=5 \mathrm{~m} ; y_{0}=0 \mathrm{~m}$, see Fig. 2 . The centerline is represented by the cubic spline with the control points at the end and two intermediate with the following coordinates $x=-2.5 \mathrm{~m}, y=1 \mathrm{~m}$ and $x=0 \mathrm{~m}, y=2 \mathrm{~m}$.

\footnotetext{
Ing. Martin Kalina.: Institute of structural mechanics, Brno University of Technology, Veveř́ 331/95; 602 00, Brno; CZ, kalina.m1@fce.vutbr.cz

** doc. Ing. Petr Frantík, Ph.D.: Institute structural mechanics, Brno University of Technology; Veveř́ 331/95; 602 00, Brno; CZ, kitnarf@centrum.cz
} 


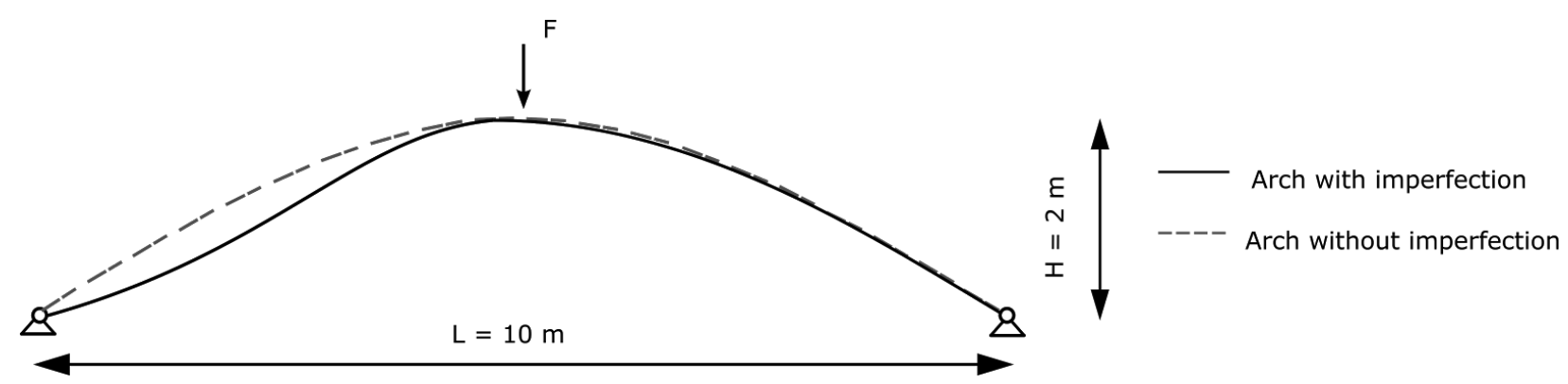

Fig. 1: Shape of the studied imperfected arch

\section{Description and computation model}

The arch is in the numerical model represented by 20 physically linear beam elements able to solve large displacements. The loading is provided by controlled displacement of top joint and follows the movement according to the selected mapping method, described in detail (Kalina, 2017). The solution is found by dynamic simulation using nonlinear dynamical system approach (Macur, 1996), which will preserve that the found state is stable equilibria. After each displacement step of the top node, its position, and appropriate potential energy of the model are recorded from which the map of the surface is made.

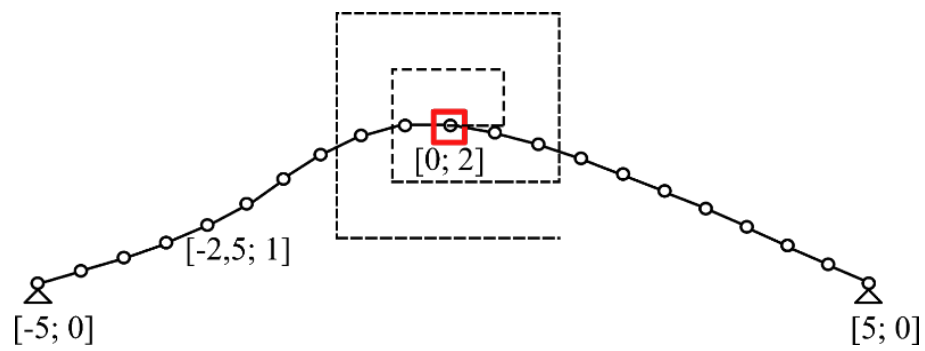

Fig. 2: Model with marked movement of the top node on behalf of the chosen mapping method

\section{The existence of static equilibrium states of a beam model}

During mapping of potential energy surface expected behaviour of the modelled arch was observed. For sake of simple visualization, we can take a look on case with conservated vertical displacement $y=1.640969 \mathrm{~m}$. When crossing from point $x=-0.20318 \mathrm{~m}$, to point $x=-0.19068 \mathrm{~m}$, there is a jump to another state. see Fig. 3. It was assumed that it happened due to transition from static equilibrium state into the other one. Therefore, remarkable change of potential energy of the model takes place. Thus, the shape of the arch can appear in two forms at the same time. Better view of the situation can be achieved through energetic surface which was studied when the model was in first static state and in second state. In Fig. 3 be seen the change of potential energy of the model for individual horizontal coordinate of the top joint. 


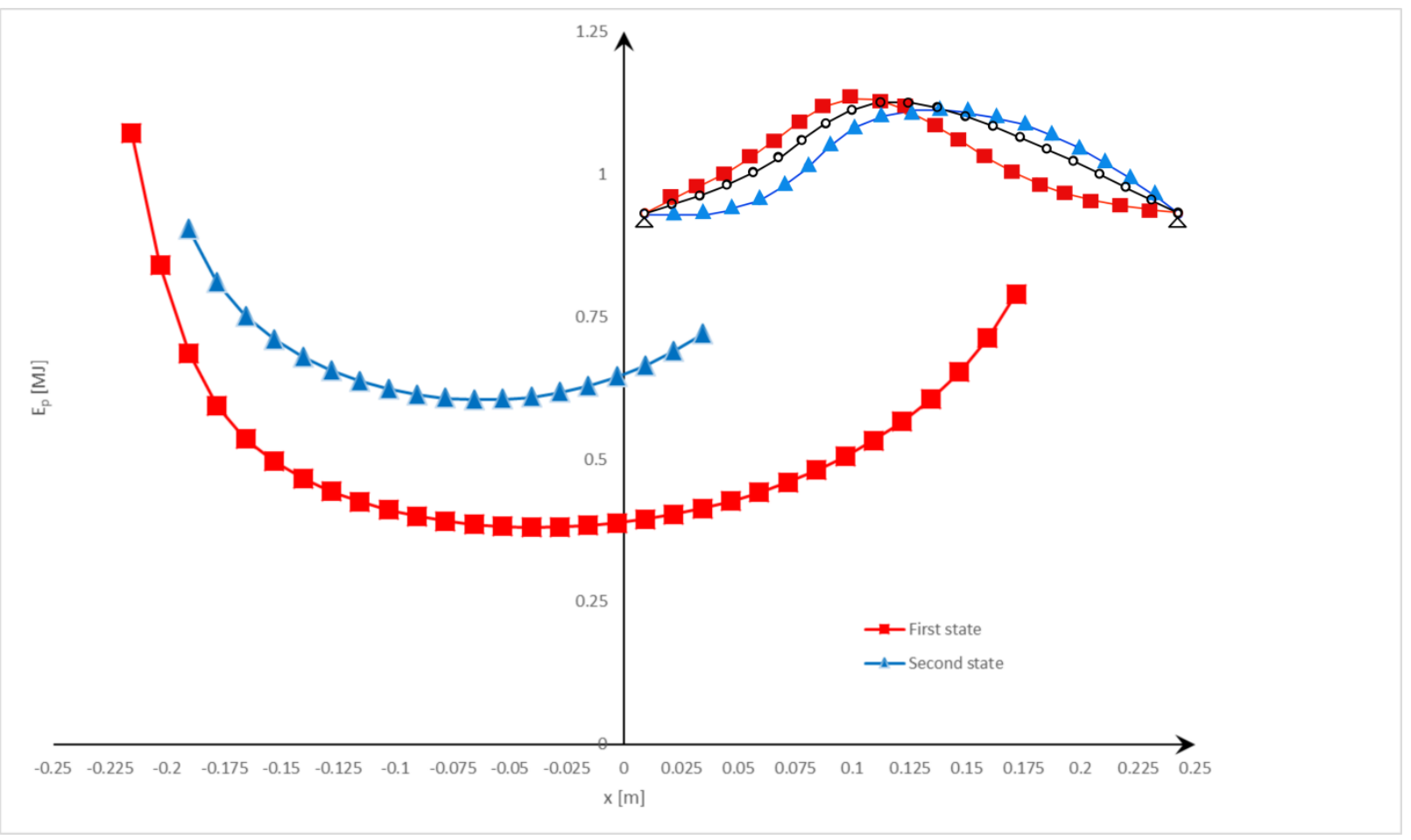

Fig. 3: Function of potential energy of the model for two different static equilibrium states with fixed vertical coordinate of the loading point $y=1.640969 \mathrm{~m}$.

\section{Searching for the static equilibrium states occurrence}

First of all, it was necessary to find the point of transition of one state into another one. Thus, the determination of situation of the bifurcation point of the model is concerned. By the movement control of the top joint within the application MapPoEng, the approximate coordinate of the bifurcation point will be got to. The step of displacement of top joint, and subsequent displacement to the point of bifurcation point were minimized up to the moment until they were stabilized at the values of coordinates $x=-0.044708959 \mathrm{~m} ; y=-0.25 \mathrm{~m}$. In Fig. 3., there is presented a schematic illustration of the model in original state, and in two postcritical ones: in original state (black colour), in the first state (red colour), and in the second state (blue colour).

The search for energetic surface $\mathrm{E}_{\mathrm{pi}(\mathrm{x}, \mathrm{y})}$ of particular static state is obtained by the method named "Diagonal Base Comb", presented in Fig. 4. The method moves the top joint in the marked trajectories originated from the origin. After each step to neighbouring grid cell, it records the value of potential energy. If the end of particular trajectory is achieved, method returns to the point with the last recorded potential energy (diagonal point) and goes on in another direction.

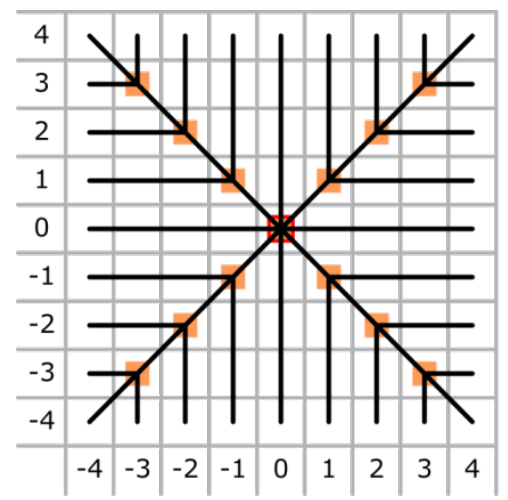

$$
\begin{aligned}
& \text { — path of the loading point } \\
& \square \text { origin } \\
& \square \text { restoring the state }
\end{aligned}
$$

Fig. 4: Illustration of the method DiagonalBaseComb

For recording the potential energy of each particular state, the coordinate of the top joint is positioned closely to the bifurcation point, and the simulation is started. The output is represented by rectangular grid 
of samples of values of potential energies within coordinate's $\mathrm{x}$ and $\mathrm{y}$ raster. To distinguish between individual states from each other, the obtained values $\mathrm{V}_{(\mathrm{x}, \mathrm{y})}$ will be expressed in percents:

$$
V_{(x, y)}=\frac{-E_{p 1(x, y)}+E_{p 2(x, y)}}{E_{p 2(x, y)}} \cdot 100[\%]
$$

In Fig. 5, the colour spectrum of the values of potential energies of the whole model in the coordinate's $x$ and y raster is seen. From the results, it can be determined in which state the model for the given coordinate occurs (blue - the first state, green - the second state). The residue of the colour spectrum indicates a continuous transition from one state to another one.

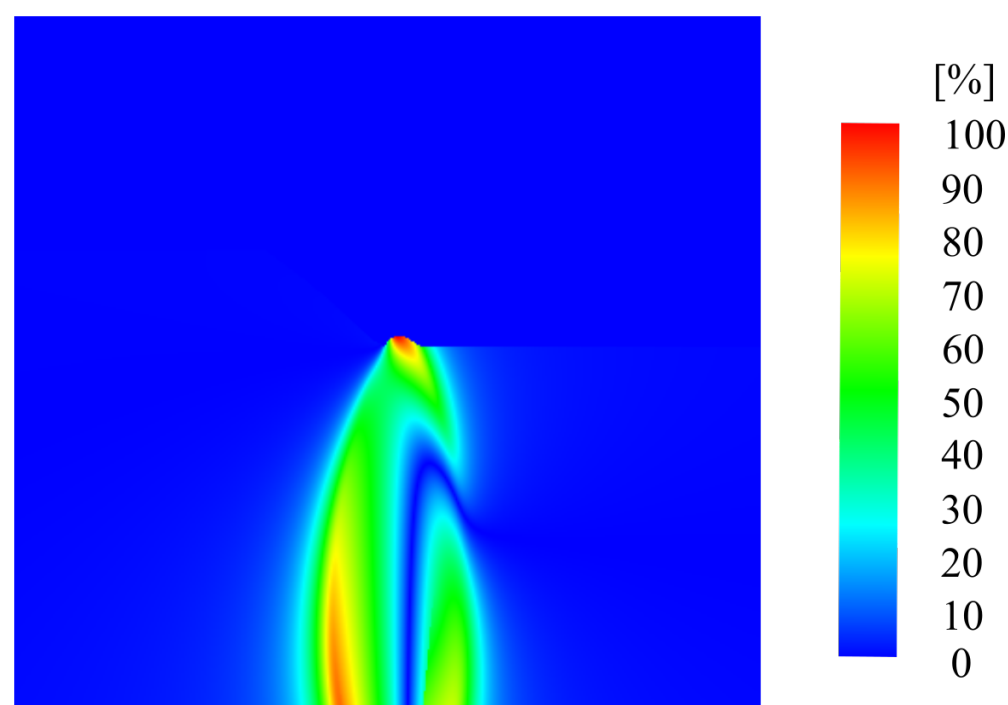

Fig. 5: Illustration of energetic surface of the whole model

\section{Conclusions}

The present contribution dealt with the postcritical states of arch modelled as a curved rod with geometrical imperfection. It was found at the simulation that the model had at least two static equilibrium states at loading. These states were detected by calculating the energetic surface and finding the bifurcation point. The fact that at least two equilibrium states arise in the model during loading, helps us to understand this behaviour. When modelling such a structure, it is necessary to remember that the model need not deform only by one way, but that the second deformation state can occur.

\section{Acknowledgement}

The paper was realized within the frame of solution of the project GAČR 17-01589S.

\section{References}

Dinkler, D. Pontow, J. (2006) A model to evaluate dynamic stability of imperfection sensitive shells, Computational Mechanics, 37, 6, pp 523-529.

Frantík, P. (2007) Simulation of the stability loss of the von Mises truss in an unsymmetrical stress state, Journal Engineering Mechanics, 14, 3, pp. 155-162.

Frantík, P. (2017) MapPoEng, http://mappoeng.kitnarf.cz.

Kalina, M. (2016) Stability problems of pyramidal von Mises planar truss with geometrical imperfection, International Journal of Theoretical and Applied Mechanics, 1, pp. 118-123.

Kalina, M. (2017) Mapping of static equilibrium states of arched structure, In: International conference Modelling in Mechanics 2017, ISBN 978-80-248-4010-9.

Macur, J. (1996) Úvod do teorie dynamických systémů a jejich simulace, VUT Brno, ISBN 80-214-0698-4

Timoshenko, S. P. and Gere, J. M. (1961) Theory of Elastic Stability, 2 ed., McGrawl-Hill. 